\title{
Minireview
}

\section{A Biomarker-Centric Approach to Drug Discovery and Development: Lessons Learned from the Coronavirus Disease 2019 Pandemic}

\author{
Kelly A. Fader, ${ }^{1}$ Jiangwei Zhang, ${ }^{1}$ Joseph P. Menetski, Ravi I. Thadhani, Elliott M. Antman, \\ Gary S. Friedman, Shashi K. Ramaiah, and Vishal S. Vaidya
}

Worldwide Research, Development and Medical, Pfizer Inc., Cambridge, Massachusetts (K.A.F., J.Z., G.S.F., S.K.R., V.S.V.); Foundation for the National Institutes of Health, Bethesda, Maryland (J.P.M.); Mass General Brigham, Boston, Massachusetts (R.I.T.); and Brigham and Women's Hospital, Harvard Medical School, Boston, Massachusetts (E.M.A.)

Received July 6, 2020; accepted October 13, 2020

\begin{abstract}
Faced with the health and economic consequences of the global spread of severe acute respiratory syndrome coronavirus 2 (SARS-CoV-2), the biomedical community came together to identify, diagnose, prevent, and treat the novel disease at breathtaking speeds. The field advanced from a publicly available viral genome to a commercialized globally scalable diagnostic biomarker test in less than 2 months, and first-inhuman dosing with vaccines and repurposed antivirals followed shortly thereafter. This unprecedented efficiency was driven by three key factors: 1) international multistakeholder collaborations, 2) widespread data sharing, and 3) flexible regulatory standards tailored to meet the urgency of the situation. Learning from the remarkable success achieved during this public health crisis, we are proposing a biomarkercentric approach throughout the drug development pipeline.
\end{abstract}

\section{Introduction}

On December 31, 2019, the China Country Office of the World Health Organization (WHO) was notified of a "pneumonia of unknown cause" in the city of Wuhan (World Health Organization, 2020 January 5). Severe acute respiratory syndrome coronavirus 2 (SARS-CoV-2), the previously unknown virus responsible for this disease, has since spread to more than 180 countries worldwide, resulting in the greatest global health crisis, thus far, of the 21st century. The biomedical community's response to this unprecedented pandemic has taught us that when all stakeholders join forces for

This paper received no external funding.

Fader, Zhang, Friedman, Ramaiah, and Vaidya are employees of Pfizer Inc.

${ }^{1}$ K.A.F. and J.Z. contributed equally to this work.

https://doi.org/10.1124/jpet.120.000204.
Although all therapeutic areas would benefit from end-to-end biomarker science, efforts should be prioritized to areas with the greatest unmet medical needs, including neurodegenerative diseases, chronic lower respiratory diseases, metabolic disorders, and malignant neoplasms.

\section{SIGNIFICANCE STATEMENT}

Faced with the unprecedented threat of the severe acute respiratory syndrome coronavirus 2 pandemic, the biomedical community collaborated to develop a globally scalable diagnostic biomarker (viral DNA) that catalyzed therapeutic development at breathtaking speeds. Learning from this remarkable efficiency, we propose a multistakeholder biomarker-centric approach to drug development across therapeutic areas with unmet medical needs.

ABBREVIATIONS: BGI, Beijing Genomics Institute; CDC, Centers for Disease Control and Prevention; COVID-19, coronavirus disease 2019; DDT, drug development tool; EUA, Emergency Use Authorization; FDA, Food and Drug Administration; IND, Investigational New Drug; IVD, in vitro diagnostic; NASH, nonalcoholic steatohepatitis; NIH, National Institutes of Health; RT-PCR, reverse-transcription polymerase chain reaction; SARS-CoV, severe acute respiratory syndrome coronavirus; WHO, World Health Organization. 
comparison with other highly infectious respiratory disease outbreaks. During the 2002-2004 epidemic, which involved the closely related SARS-CoV-1 virus, it took nearly 6 months to design a molecular test for diagnosing the infection (Drosten et al., 2003). In contrast, development, approval, and distribution of SARS-CoV-2 diagnostic assays have progressed at breakneck speeds (Sheridan, 2020). A key factor driving this unprecedented efficiency was alignment within the biomedical community, not only between various stakeholders but also across international borders (Fig. 1). Within a week of the disease being reported, an international team of academic research laboratories, hospitals, and public health institutes across China and Australia successfully identified the novel SARS-CoV-2 virus using a sample of bronchoalveolar lavage fluid (Wu et al., 2020). Shortly after the genome sequence was deposited in GenBank, researchers at the Charité University Hospital of Berlin, in collaboration with academic laboratories in China and Europe, published the first real-time reversetranscription polymerase chain reaction (RT-PCR)-based diagnostic test (Corman et al., 2020). Regulatory agencies such as the U.S. Food and Drug Administration (FDA) granted Emergency Use Authorization (EUA) to commercial laboratories [e.g., Beijing Genomics Institute (BGI)] and public health institutes [e.g., U.S. Centers for Disease Control and Prevention (CDC)], permitting rapid production of this and similar kits. To meet the overwhelming need for large-scale testing, BGI opened an emergency response laboratory in Wuhan, processing over 10,000 samples per day. By February 6, 2020, the WHO had distributed 250,000 diagnostic kits to 159 laboratories around the world, facilitating rapid testing and public health risk assessment. Initial testing in the United States was undertaken by the CDC, but commercial laboratories, including LabCorp, Roche, and Abbott Laboratories, soon joined in with their own diagnostic assays (Sheridan, 2020).

Beyond diagnostic testing, development of vaccines and antivirals against SARS-CoV-2 has also relied on coordination between government agencies, clinicians, and the private sector (Fig. 1). For example, the mRNA-1273 vaccine is a joint effort between the U.S. National Institutes of Health (NIH) and the biotechnology company Moderna. After jointly designing the mRNA sequence, Moderna began manufacturing the vaccine while the NIH led the phase 1 safety/tolerability trial. On May 6, 2020, the FDA approved the Investigational New Drug (IND) application for mRNA-1273, allowing the vaccine to proceed to a phase 2 trial in 600 people. Several other public-private partnerships have also been formed to promote a coordinated international strategy for vaccine and antiviral research, including the Accelerating COVID-19 Therapeutic Interventions and Vaccines (ACTIV) partnership, which consists of more than 12 pharmaceutical companies along with government agencies (NIH, FDA, CDC, U.S. Department of Health and Human Services, European Medicines Agency) and the nonprofit Foundation for the National Institutes of Health (Collins and Stoffels, 2020). Meanwhile, Biogen Inc., the Broad Institute of Massachusetts Institute of Technology and Harvard, and Mass General Brigham have formed a multistakeholder consortium that will build and share a first-of-its-kind COVID-19 biobank consisting of blood samples and biologic/medical data from Massachusetts-based volunteers (Biogen Inc, 2020). Access to this localized cluster of patient samples and data not only will allow researchers to gain novel insight into SARS-CoV-2 biology and behavior but also may guide the development of therapeutic treatment options.

Transparent and robust data sharing is another important element that distinguishes the scientific response to COVID-19 from previous global health crises (Heymann, 2020; Xu et al., 2020). Sharing laboratory, epidemiologic, and clinical data not only supported the development of diagnostic assays and vaccines but also guided public health decision-making in real time. Scientific journals (e.g., Nature Human Behavior) and publishers (e.g., Springer Nature) mobilized their resources to expedite the peer review and publication of studies specifically related to the COVID-19 pandemic, along with the immediate release of associated data, code, and protocols (Science in the time of COVID-19., 2020). This allowed time-critical information to be globally disseminated while still vetting the content and attributing the work to those responsible. Notably, the polymerase chain reaction-based diagnostic assay by Corman et al. (2020) was submitted to Eurosurveillance on January 21, accepted on January 22, and published online on January 23, 2020. The biomarker genes targeted in this assay, as well as the sequence of the mRNA-1273 vaccine, were identified using the novel SARS-CoV-2 genome, which was made publicly available through open-access data bases such as GenBank and Global Initiative on Sharing All Influenza Data (Sheridan, 2020). Moreover, several leading scientific journals including The New England Journal of Medicine, The Journal of the American Medical Association, and The Journal of Infectious Diseases made their COVID-19-related content freely available to the public, including primary research articles, clinical reports, commentaries, instructional videos, and podcasts. In an unprecedented effort to share data between normally competing private-sector corporations, top pharmaceutical and biotechnology leaders have organized the COVID Research and Development consortium. This industry collaboration enables prioritization of resources and clinical testing for therapeutics and vaccines with the greatest chance of success, accelerating drug availability for patients and reducing redundancy in the field (Fishburn, 2020, April 15).

The authority of federal regulatory agencies to grant streamlined approval was also crucial in enabling a timely response. Under emergency circumstances, EUA from the FDA allows previously unapproved medical products or unapproved uses of approved products to be employed for the diagnosis, treatment, or prevention of life-threatening conditions when no viable alternatives are available (U.S. Food and Drug Administration, 2020). Once the scope of the SARSCoV-2 outbreak became evident, the FDA worked with both the CDC and the private sector to expedite the review of urgently needed diagnostic tests, approving their use in as little as 1 day. Expedited review was also applied to IND applications for novel vaccines and repurposed antivirals, greatly accelerating drug availability for patients. For example, 2 days after the clinical trial demonstrated promising results, the FDA issued EUA for Gilead's antiviral remdesivir as a potential COVID-19 treatment of patients hospitalized with severe disease. Although EUA is the exception rather than the norm, the FDA's response to SARS-CoV-2 has 


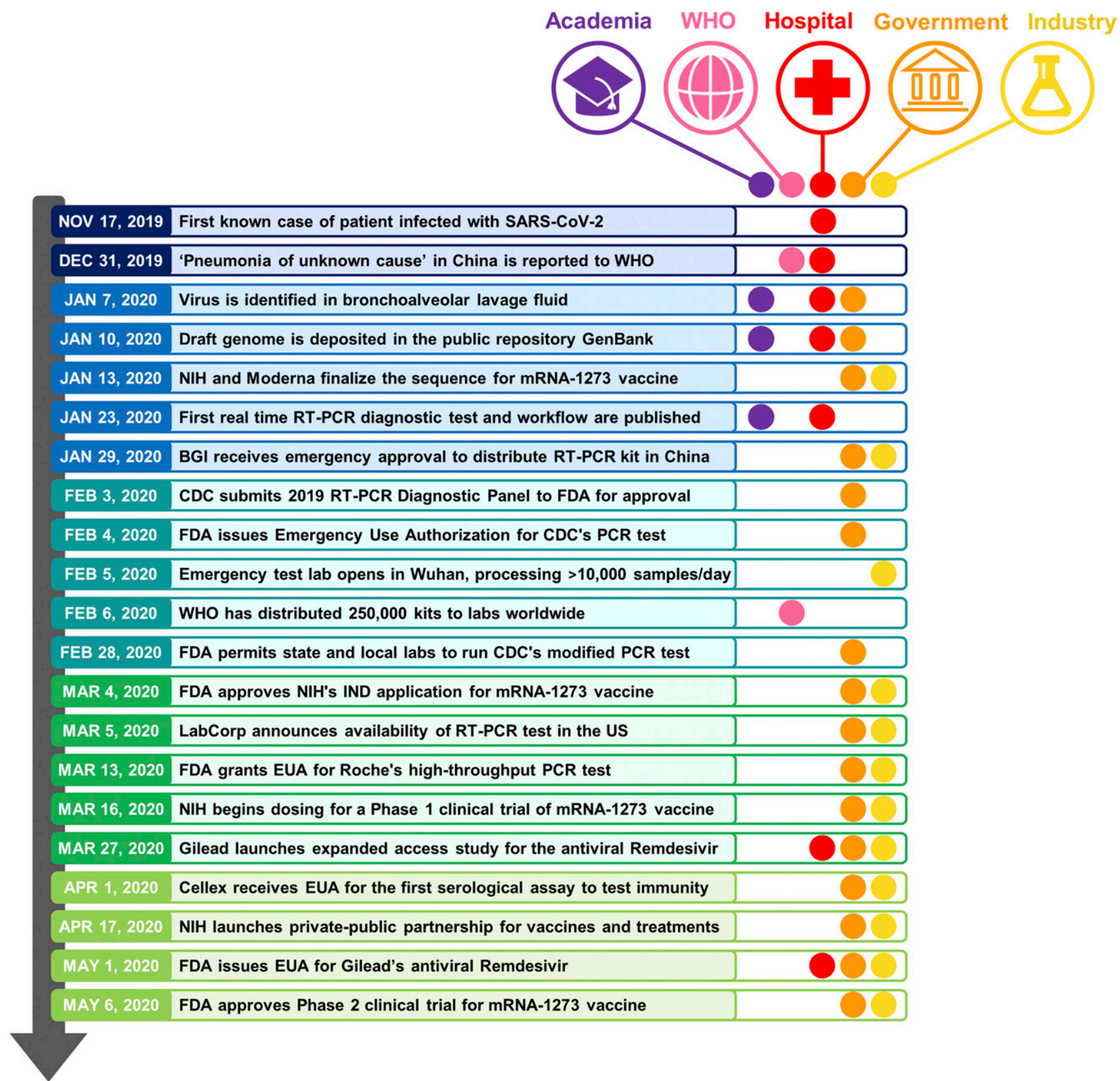

Fig. 1. Multistakeholder international collaborations enable acceleration of scientific breakthroughs to manage the COVID-19 pandemic. During the COVID-19 pandemic, the biomedical community came together to identify, diagnose, prevent, and treat the novel SARS-CoV-2 virus at breakneck speeds. This was accomplished through international precompetitive multistakeholder collaborations, as illustrated by the color-coded dots to the right of each milestone. Development of a diagnostic biomarker for the viral infection was the catalyst that drove this unprecedented speed, efficiency, and success.

demonstrated the importance of flexible regulatory pathways tailored to match the severity of the hazard. The imminent threat of the global SARS-CoV-2 outbreak undoubtedly instilled a sense of urgency within all stakeholders. Under emergency circumstances, necessity often drives creative problem solving and innovative solutions, accelerating scientific breakthroughs. However, it should not take a pandemic to facilitate this degree of creativity and efficiency in drug development.

\section{Previous International Disasters Informed SARS-CoV-2 Emergency Preparedness}

Prior viral outbreaks [SARS-CoV-1 (2002-2004), Middle East respiratory syndrome (MERS, 2012), and Ebola
(1989-2019)] and other public health catastrophes have contributed to the evolution of the WHO Global Outbreak Alert and Response Network, as well as the collaboration between the CDC and the U.S. Agency for International Development Emerging Pandemic Threats program (Lucchini et al., 2017; Ando, 2018). With the intention of preempting zoonotic disease outbreaks and/or mitigating their impacts, these organizations provide stakeholders with an existing framework to leverage advances in pathogen biology, genomics, bioinformatics, machine learning, and near-realtime epidemiologic data. In 2016, the U.S. National Security Council developed the Playbook for Early Response to HighConsequence Emerging Infectious Disease Threats and Biological Incidents, which serves as a decision-making tool for 
the government by outlining 1) questions to ask, 2) which agencies to consult, and 3) key decisions that may require deliberation (National Security Council, 2016). As a result of these emergency preparedness plans, the biomedical community was able to work with regulatory agencies to expedite recruitment for observational and interventional clinical trials related to SARS-CoV-2 therapeutics and diagnostics. Moving forward, lessons learned from the COVID-19 pandemic, overlaid upon emergency preparedness plans from prior public health crises, could be leveraged by private and public stakeholders to coordinate a rapid response to emerging diseases that currently lack optimal biomarkers.

\section{End-to-End Biomarker Science to Accelerate Drug Discovery and Development}

Development of specific, sensitive, and reliable biomarkers catalyzed the rapid response to COVID-19. Per the FDA, a biomarker is a "defined characteristic that is measured as an indicator of normal biologic processes, pathogenic processes, or responses to an exposure or intervention, including therapeutic interventions." Based on their intended use, biomarkers can be classified into one of seven Biomarkers, EndpointS, and other Tools (BEST) categories: susceptibility/risk, diagnostic, and prognostic biomarkers measure the presence or status of the disease, whereas monitoring, predictive, pharmacodynamic/response, and safety biomarkers measure response to treatment (FDA-NIH Biomarker Working Group, 2016). Throughout the COVID-19 pandemic, two key diagnostic biomarkers have been used to identify cases of the novel disease and track its spread worldwide. First and foremost, specific sequences of viral SARS-CoV-2 DNA within patient nasopharyngeal or sputum samples formed the basis for the RT-PCR in vitro diagnostic (IVD) assays used by the WHO, public health agencies (e.g., CDC), and commercial laboratories. Secondly, anti-SARS-CoV-2 antibodies (neutralizing or total antibodies) detected through serological tests are used to determine whether an individual has previously been infected with the virus. The ability to diagnose cases of the SARS-CoV-2 infection paved the way for understanding disease progression and developing treatment options. For example, clinical evaluation of patients with COVID-19 revealed that elevated levels of interleukin-6, interleukin-10, interferon $\gamma$-inducible protein 10 (IP-10), and C-reactive protein (CRP) correlate with disease severity and length of hospitalization (Laing et al., 2020; Luo et al., 2020). These cytokines and inflammatory markers therefore serve as prognostic biomarkers for COVID-19, which could be used to monitor disease progression and predict adverse outcomes. Beyond immune and inflammatory responses, clinical studies have identified biomarkers that correlate with several other biologic responses of COVID-19, including hematologic changes (e.g., basophil and plasmacytoid dendritic cell depletion, neutrophil-to-lymphocyte ratio), coagulation (e.g., D-dimer, prothrombin time), and effector organ dysfunction (e.g., creatine kinase, aspartate aminotransferase, and troponin) (Laing et al., 2020; Ponti et al., 2020a). Moreover, homocysteine recently emerged as a novel predictor of cardiovascular complications caused by COVID-19, allowing classification of patients based on risk of vascular damage and thromboembolism (Ponti et al., 2020b). The unparalleled pace of the pandemic response demonstrates the value of adopting a biomarker-centric approach to drive rational management and evidence-based treatment of complex human diseases. However, it is important to note that our understanding of COVID-19 continues to evolve, and thus only time will reveal the true diagnostic and prognostic performance of these novel biomarkers, along with the long-term effects of the disease.

In the case of a highly infectious respiratory disease such as COVID-19, diagnostic biomarkers are the initial priority. However, biomarkers can be strategically used throughout the entire drug development pipeline, resolving key issues as they arise (Fig. 2A). Pharmacodynamic/response biomarkers can build confidence in the drug by demonstrating target binding and modulation of pharmacologic activity. Based on the riskbenefit profile of the lead candidate, safety biomarkers can be deployed for monitorability and reversibility in preclinical studies and first-in-human trials. Proof of concept in phase $1 \mathrm{~b}$ or 2 trials can be achieved by demonstrating change in disease monitoring biomarkers. To optimize efficacy and mitigate safety concerns, biomarkers can be used to enrich the clinical trial population through the following strategies: 1) decreased heterogeneity using diagnostic biomarkers, 2) enrichment based on the likely outcome of the disease using prognostic biomarkers, and 3) enrichment based on therapeutic intervention using predictive biomarkers (Antman and Loscalzo, 2016). Biomarker-driven innovative clinical trial design is also favored by regulatory agencies, not only because it facilitates evidence-based decision-making but also because it serves the interests of patients. Lastly, biomarker data can be continually collected, even after regulatory approval, to generate realworld evidence for safety surveillance and expanded use. Strategic biomarker use throughout drug development offers several benefits, including optimized efficiency, enhanced productivity, and increased probability of clinical success. Ultimately, end-to-end biomarker science not only accelerates evidence-based drug development but also provides novel insight into disease pathogenesis and enables novel therapeutic discovery.

Development of omics technology has revolutionized the field of biomarker science. High-throughput techniques such as transcriptomics, proteomics, and metabolomics enable rapid and methodical discovery of novel biomarkers, as they allow for comprehensive untargeted molecular profiling of preclinical and clinical samples. The cost of these technologies has drastically decreased in recent years, and in turn, the number of publications identifying candidate biomarkers has skyrocketed (Poste, 2011; Park and Kim, 2016). However, only a fraction develop into robust and validated biomarkers that can be reliably deployed in drug development, with fewer than $0.1 \%$ of reported candidate biomarkers successfully transitioning to become routine clinical assays (Poste, 2011; Bossuyt, 2014; Goossens et al., 2015). This is not entirely surprising, as analytical/clinical validation and regulatory acceptance require large, time-consuming, expensive studies, which are often not feasible for an academic research group to undertake. Moreover, discovery is typically performed on "samples of convenience," which may not accurately reflect the patient population in which the biomarkers are expected to be used (Ransohoff and Gourlay, 2010; Simon, 2011). This may introduce unrecognized confounding variables leading to false-positive associations that cannot be replicated. Although the onset of omics profiling has expedited candidate 
A

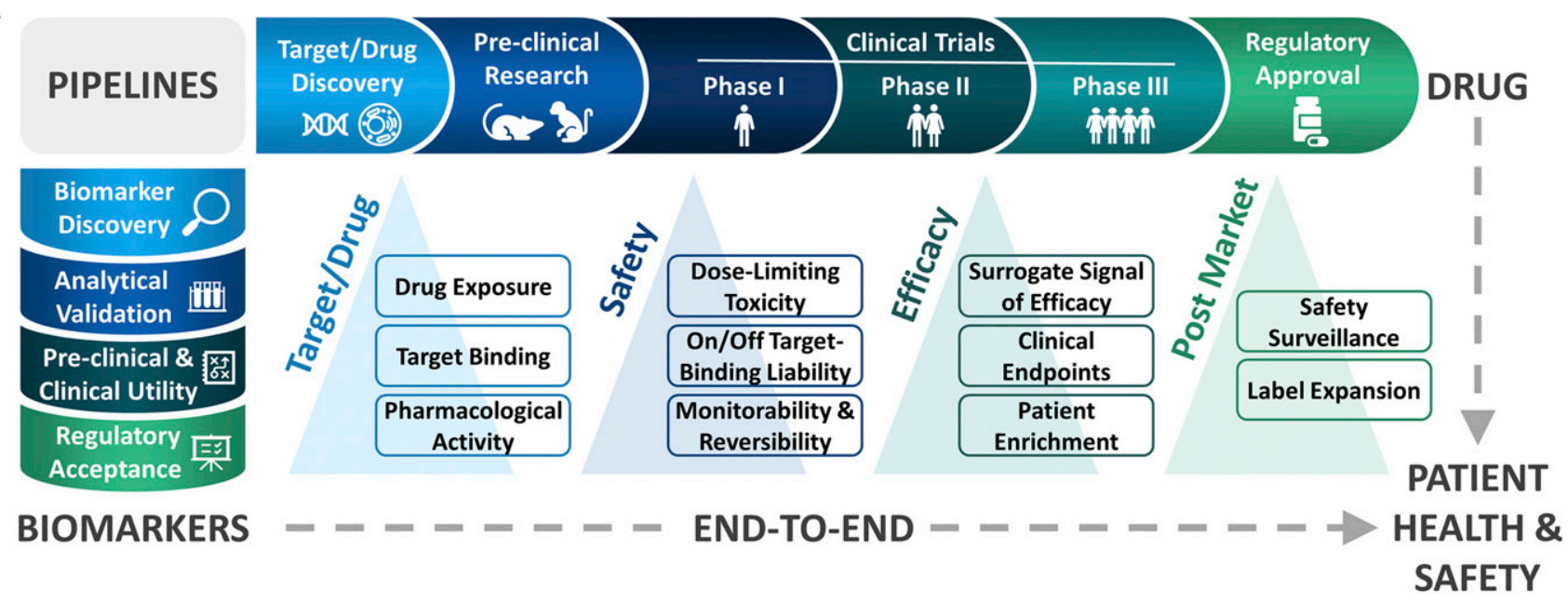

B

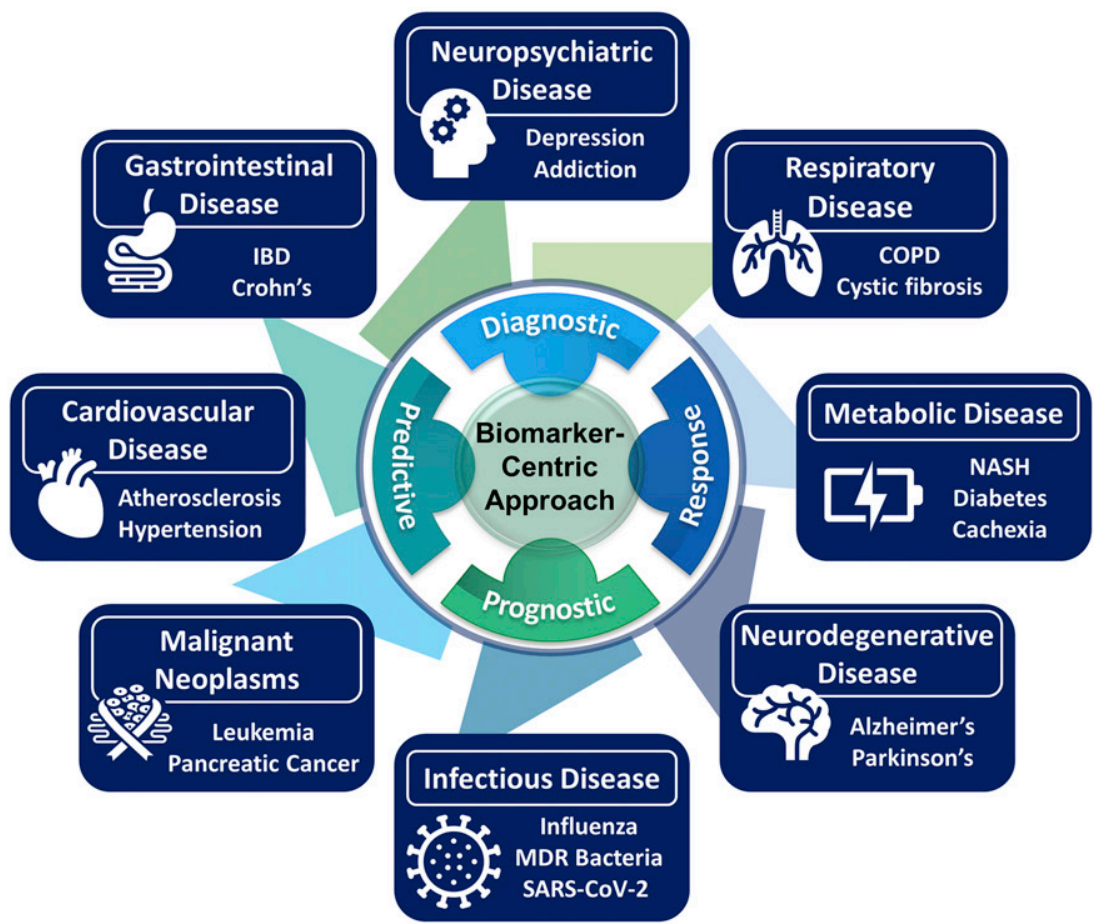

Fig. 2. End-to-end biomarker strategy to accelerate drug development for unmet medical needs. (A) To maximize the probability of clinical success, a biomarker-centric approach should be adopted during drug development. Biomarkers can be strategically used throughout the drug development pipeline, from initial assessment of target engagement, to safety, efficacy, and finally postmarket approval. Before a novel biomarker can be used to influence regulatory decision-making within a drug development program, it must undergo analytical validation, preclinical or clinical utility studies, and regulatory acceptance. This multistep process closely resembles the phases of the drug development pipeline itself. (B) In an effort to prioritize therapeutic areas that would benefit from a biomarker-centric approach, eight focus areas were identified based on mortality rate, impact on population health, and unmet medical needs. COPD, chronic obstructive pulmonary disease; IBD, inflammatory bowel disease; MDR, multidrug resistant.

biomarker discovery, the progress of validation, qualification, and clinical implementation lag behind. A coordinated effort among stakeholders is required to push beyond biomarker discovery, engaging experts from academic medical centers, the pharmaceutical industry, regulatory authorities, and patient advocacy groups.

The sudden burden of SARS-CoV-2 on public health and the global economy synergistically motivated the spectrum of stakeholders to join forces for the development of biomarkers, therapeutics, and vaccines. This collaborative momentum should be maintained as we move beyond the pandemic and turn our attention back to the world's most impactful diseases-diseases which may actually dwarf the incidence and mortality rates observed with COVID-19. To integrate our efforts and focus our resources, the biomedical community must prioritize the top unmet medical needs, which urgently require novel therapeutics. From a public health perspective, we have identified eight therapeutic focus areas that would benefit most from a biomarker-centric approach throughout drug development: chronic lower respiratory diseases, neuropsychiatric diseases, cardiovascular disease, malignant neoplasms, neurodegenerative diseases, metabolic disorders, infectious diseases, and gastrointestinal diseases (Fig. 2B). Prioritization was based on these therapeutic areas meeting at least two of the following three criteria: 1) ranked in the top 10 leading causes of death in the United States in 2017 
(Heron, 2019), 2) ranked in the top 10 most impactful conditions on the United States population according to Blue Cross Blue Shield's Health Index (Blue, 2020), and 3) identified as a top unmet medical need (Scavone et al., 2019). Although a comprehensive end-to-end biomarker approach is ideal, these focus areas differ in the type of biomarker most urgently needed at the present time. For example, as we strive to apply precision medicine to the field of oncology, predictive biomarkers are needed to stratify patient populations, guide therapy selection, and design multidrug chemotherapy programs. In the case of nonalcoholic fatty liver disease, a liver biopsy is needed to definitively diagnose a patient's stage along the spectrum, as imaging techniques cannot detect hepatic inflammation or fibrosis. Treatment of nonalcoholic fatty liver disease would therefore benefit greatly from noninvasive diagnostic biomarkers capable of distinguishing hepatic steatosis from steatohepatitis (NASH), fibrosis, cirrhosis, and hepatocellular carcinoma. Developing novel therapeutics for neuropsychiatric and neurodegenerative disease is notoriously difficult, as clinical efficacy currently relies on subjective evaluations such as self-rated scales (e.g., Beck Depression Inventory) and neuropsychological tests that assess memory, visuospatial skills, and verbal abilities. Response biomarkers that provide an objective and quantifiable measure of clinical efficacy would be invaluable to achieving technical and regulatory success for therapeutics targeting Alzheimer's disease and depression. For patients with chronic obstructive pulmonary disease, there are currently no established clinical methods for predicting imminent exacerbations, and therefore prognostic biomarkers would be instrumental in preventing deterioration, hospitalization, and mortality with this disease. Confident diagnosis, objective clinical endpoints, and the ability to stratify patient subpopulations will substantially increase the likelihood of clinical success and market approval. Beginning with these eight focus areas, we are proposing that the biomedical community adopts a biomarker-driven strategy for drug development, as demonstrated during the SARS-CoV-2 outbreak. Successful implementation of this strategy will depend on 1) precompetitive multistakeholder collaborations, 2) widespread data sharing with detailed metadata, and 3) flexible, evidencebased regulatory acceptance.

\section{Implementation of a Biomarker-Centric Strategy Based on Lessons Learned during a Global Pandemic}

To tackle the unmet medical needs of the world, the biomedical community must work together to validate, qualify, and implement novel biomarkers that accelerate drug development. As demonstrated during the COVID-19 pandemic, precompetitive collaborations between multiple stakeholders are fundamental to achieving timely results. Beyond the pharmaceutical industry itself, collaborative drug development efforts should also include academia, teaching hospitals, biobanks, commercial laboratories, contract research organizations, and government agencies. Each stakeholder possesses unique strengths that should be considered when dividing and delegating tasks across the biomarker pipeline. Academic research laboratories excel in exploratory research, enabling scientific curiosity and innovation. The pharmaceutical industry not only is focused on identifying areas of need and answering patient-specific questions but also possesses the resources to carry out large-scale validation studies themselves or contract them out to a contract research organization. With access to well annotated clinical samples across different disease stages, teaching hospitals and biobanks can initiate or support biomarker discovery and validation studies, whereas commercial laboratories typically focus on developing and distributing assays for preclinical and clinical use. Finally, government agencies such as the FDA set standards and requirements, oversee the Biomarker Qualification Program, and serve as a system of checks and balances. Acknowledging the strengths and limitations of each stakeholder will transform biomarker development into a cooperative rather than competitive venture. Several multistakeholder teams and consortia have been formed to address unmet biomarker needs in specific therapeutic areas. This has led to the recent emergence of many success stories involving pharmaceutical implementation of novel biomarkers during drug development and acceptance/qualification of these biomarkers by regulatory authorities. Representative examples of successful biomarker programs are listed in Fig. 3 to illustrate how collaborative efforts in the biomarker field can accelerate the development of drugs for complex diseases such as Alzheimer's disease, cancer, and fungal infections.

The success of a candidate biomarker is largely dependent on the quality and suitability of the samples selected during the discovery phase. At the time of publication, large omics data sets are expected to be deposited into public data bases such as GenBank, Gene Expression Omnibus, and the $\mathrm{Na}$ tional Metabolomics Data Repository. Transparency, completeness, and accuracy are therefore essential for ensuring these publicly available data sets and clinical trial reports can be strategically mined for valuable information. Moreover, detailed and standardized metadata allow for careful consideration during sample selection and statistical analysis, ensuring the discovery population is comparable to the intended use population. Minimum reporting guidelines have been outlined for various omics data types submitted to public repositories including microarrays (MIAME: Minimum Information About a Microarray Experiment), nucleotide sequencing (MINISEQE: Minimum Information About a High-throughput Nucleotide Sequencing Experiment), proteomics (MIAPE: Minimum Information About a Proteomics Experiment), and metabolomics (CIMR: Core Information for Metabolomics Reporting; MSI: Metabolomics Standards Initiative) (Misra et al., 2018). Similarly, clinical reports of diagnostic, observational, and prognostic studies should adhere to the STARD (Standards for Reporting of Diagnostic Accuracy Studies), STROBE (Strengthening the Reporting of Observational Studies in Epidemiology), and REMARK (Reporting Recommendations for Tumor Marker Prognostic Studies) guidelines, respectively (Vandenbroucke, 2009; Johansen and Thomsen, 2016). Widespread data sharing in the precompetitive space drives scientific progress, which is in the best interest of each stakeholder, especially the patients.

In the early stages of drug development, exploratory biomarkers can be used to internally guide target/drug discovery and preclinical research without requiring external approval. In contrast, biomarkers that will impact regulatory 


\begin{tabular}{|c|c|c|c|}
\hline DISEASE & COLLABORATION & BIOMARKER SUCCESS & REFERENCE \\
\hline \multirow[t]{2}{*}{ Alzheimer's Disease } & $\begin{array}{l}\text { Alzheimer's Disease } \\
\text { Neuroimaging Initiative } \\
\text { (ADNI) }\end{array}$ & $\begin{array}{l}\text { Discovered tau positron emission tomography (PET), which } \\
\text { could be used as a surrogate endpoint for monitoring clinical } \\
\text { efficacy of novel treatments }\end{array}$ & $\begin{array}{l}\text { Petersen et al., } 2010 \\
\text { Weiner et al., } 2017 \\
\text { Saint-Aubert et al., } 2017\end{array}$ \\
\hline & $\begin{array}{l}\text { Washington University } \\
\text { School of Medicine \& } \\
\text { Alzheimer's Association }\end{array}$ & $\begin{array}{l}\text { Developed a mass spectrometry-based method for quantifying } \\
\text { circulating levels of phosphorylated tau ( } p \text {-tau217), allowing } \\
\text { blood-based monitoring of disease progression in clinical trials }\end{array}$ & Barthelemy et al., 2020 \\
\hline Breast Cancer & $\begin{array}{l}\text { FNIH Biomarkers } \\
\text { Consortium }\end{array}$ & $\begin{array}{l}\text { Launched the I-SPY } 2 \text { trial, which helped advance } 6 \text { novel } \\
\text { therapies (e.g. neratinib) by incorporating biomarker analysis } \\
\text { early in phase II testing }\end{array}$ & Menetski et al., 2019 \\
\hline Invasive Aspergillosis & $\begin{array}{l}\text { Mycoses Study Group } \\
\text { (MSG) }\end{array}$ & $\begin{array}{l}\text { Discovered that the fungal wall component galactomannan can } \\
\text { be used as a diagnostic biomarker, qualified by the U.S. FDA, } \\
\text { when measured in serum or bronchoalveolar lavage fluid }\end{array}$ & $\begin{array}{l}\text { Patterson, } 2011 \\
\text { Mercier et al., } 2018\end{array}$ \\
\hline
\end{tabular}

Fig. 3. Collaborative biomarker programs that have accelerated drug development. Driven by multistakeholder teams and consortia, these representative biomarker programs have advanced drug development through implementation of novel biomarkers in pharmaceutical programs and/or qualification of these biomarkers by regulatory authorities. Citations: Petersen et al. (2010); Patterson (2011); Saint-Aubert et al. (2017); Weiner et al. (2017); Mercier et al. (2018); Menetski et al. (2019); Barthélemy et al. (2020). ADNI, Alzheimer's Disease Neuroimaging Initiative; FNIH, Foundation for the National Institutes of Health; I-SPY 2, investigation of serial studies to predict your therapeutic response with imaging and molecular analysis 2; MSG, Mycoses Study Group; PET, positron emission tomography.

decision-making within a drug development program require close and repeated interactions with public health agencies such as the FDA. The FDA's Center for Drug Evaluation and Research oversees the regulatory acceptance of all biomarkers classified as drug development tools (DDTs), and any biomarkers intended to serve as IVD products such as COVID-19 biomarker tests (viral DNA, antibodies) or companion diagnostics are regulated by the FDA's Center for Devices and Radiologic Health. Although the specific standards for DDT biomarkers differ from those required for IVDs and companion diagnostics, flexibility has been integrated into each regulatory system to accommodate varying levels of urgency. The most common path to regulatory acceptance for a DDT biomarker involves submitting data demonstrating efficacy and/ or safety as part of the IND or Biologics License Application package. Although this route facilitates rapid approval, other stakeholders and pharmaceutical companies do not have access to the biomarker data submitted, leading to redundancy and/or underutilization of a valuable biomarker. It is therefore important to recognize the two other pathways through which regulatory acceptance can be attained for a DDT biomarker: 1) qualification and 2) scientific/community consensus. Considered the gold standard, qualification is a three-step process whereby the biomarker, for a stated context of use, has been shown to reliably support a specified manner of interpretation and application in drug development. Compared with the other pathways, the FDA's Biomarker Qualification Program requires the greatest investment of time and resources, and thus it is not entirely surprising that only eight sets of biomarkers have been formally qualified since the onset of the program in 2007 (U.S. Food and Drug Administration, 2020). In the long run, however, this pathway is beneficial to all stakeholders, as qualified biomarkers can be included within any drug program without requiring additional documentation or validation. According to a survey conducted among industry experts, the two key factors that would promote biomarker qualification are
1) prioritization of specific biomarkers that hold the greatest promise to accelerate drug availability for patients, and 2) establishment of an "evidentiary standards framework," which outlines agreed-upon levels of evidence required for specific uses (Lavezzari and Womack, 2016; Leptak et al., 2017). An alternative route of regulatory acceptance involves scientific consensus, whereby peer-reviewed publications demonstrate general community agreement of a biomarker's analytical and clinical utility. All stakeholders can support this pathway by publishing their rigorously conducted reproducible biomarker research. The opportune route of regulatory acceptance for a biomarker should be carefully considered on a case-by-case basis, balancing precompetitive scientific advancement with the need to reduce time to market.

\section{Limitations of Adopting a Biomarker-Centric Approach}

Although implementation of a biomarker-centric approach during drug development can optimize efficiency and increase probability of clinical success, it is important to be strategic about when novel biomarkers are developed and what stages would benefit most. We have highlighted examples of how biomarkers can be used throughout each phase of drug discovery and development. However, attempting to develop novel biomarkers for every phase simultaneously, simply for comprehension sake, could needlessly increase spending, consume resources, and delay time to market. Furthermore, identifying a long list of correlative biomarkers offers little benefit if they do not have a clearly defined purpose in the drug development program. It is therefore important to be precise and pinpoint specific questions that biomarkers can help answer. Typically, biomarkers are most valuable when they are directly linked to the mechanism of disease, and thus it is difficult to identify useful biomarkers for poorly defined diseases for which we lack a clear understanding of cause and effect. 
During drug approval, reliance on biomarkers and/or surrogate endpoints alone can confound therapeutic efficacy and have detrimental effects on patient health. For example, bedaquiline was granted accelerated approval for treatment of multidrug resistant Mycobacterium tuberculosis based exclusively on a surrogate endpoint involving conversion of the patient's sputum sample from positive to negative. After approval, clinical outcome assessments revealed treatment failure, excess mortality, and QT prolongation, resulting in a black box warning on the drug's label. If surrogate endpoints will be heavily relied upon during regulatory decision-making, it is important that they are closely linked to efficacy and that the presumed associations are promptly confirmed through additional trials (Avorn, 2013). When adopting a precision medicine approach, failure to sufficiently validate whether therapeutic efficacy is dependent on the status of a predictive biomarker can lead to unnecessary exclusion of markernegative patients from enriched clinical trials or targeted treatment eligibility (Hey et al., 2020).

Finally, it is important to evaluate potential sources of variability within a novel biomarker prior to implementation, not only when translating preclinical biomarkers across strains and species but also when measuring clinical parameters across diverse populations. Novel biomarkers identified in patients limited to a specific geographic region or race may not be generalizable to all populations, and thus follow-up studies should be performed to assess geographic and racial variability. For example, racial differences have been identified in cerebrospinal fluid biomarkers of neurodegeneration and cognitive impairment in which tau levels are lower in Black patients with Alzheimer's disease compared with White patients despite adjusting for demographic parameters and disease severity (Garrett et al., 2019). These limitations can be managed through strategic decision-making, preemptive planning, and meticulous validation, ensuring all stakeholders benefit from biomarker-centric drug development.

\section{Conclusions and Outlook}

The SARS-CoV-2 pandemic of 2019-2020 will undoubtedly be remembered as one of the most devastating and disruptive public health crises after the Spanish flu pandemic of 1918. The biomedical community's unified, tenacious, and timely response emerged as a bright spot during an otherwise dark time. This unprecedented biomarker-enabled success was driven by three key factors: 1) precompetitive international collaborations between stakeholders, 2) widespread data sharing, and 3) flexible regulatory standards tailored to suit the urgency of the situation. Given the urgency associated with the global spread of the deadly COVID-19 pandemic, there are certainly limitations in extending this approach to more common diseases that exhibit a lower mortality rate and are not contagious. Notably, the FDA's EUA pathway cannot and should not be universally applied across all therapeutic areas. However, we must acknowledge the flexibility that exists within federal regulatory requirements, choosing the most appropriate regulatory acceptance pathway on a case-bycase basis. Moreover, the main diagnostic biomarker used for COVID-19 was a viral nucleic acid sequence, which is relatively straightforward to develop and can be taken at face value. When applying precision medicine to the field of oncology, similar nucleic acid biomarkers can be used to detect specific mutations, facilitating subtype diagnosis and clinical trial enrichment. However, the vast majority of biomarker needs cannot be fulfilled by a nucleic acid sequence. For example, the identification of robust diagnostic biomarkers for NASH and cardiovascular disease is confounded by the multifactorial nature of the disease, and the poorly understood causal mechanisms underlying Alzheimer's disease and depression impede neurologic biomarker discovery. Nevertheless, biomarker development for these complex therapeutic areas can be successfully tackled through multistakeholder collaborations with precompetitive data sharing and flexible regulatory standards. If we can harness a biomarker-centric approach for drug development across therapeutic areas with the greatest unmet medical needs, the benefits for human health and scientific advancement would be incalculable.

\section{Acknowledgments}

We thank Morten Sogaard, Pfizer Inc., for his critical review and valuable feedback.

\section{Authorship Contributions}

Wrote or contributed to the writing of the manuscript: Fader, Zhang, Menetski, Thadhani, Antman, Friedman, Ramaiah, Vaidya.

\section{References}

Ando R (2018) Trust-what connects science to daily life. Health Phys 115:581-589. Antman EM and Loscalzo J (2016) Precision medicine in cardiology. Nat Rev Cardiol 13:591-602.

Avorn J (2013) Approval of a tuberculosis drug based on a paradoxical surrogate measure. JAMA 309:1349-1350.

Barthélemy NR, Horie K, Sato C, and Bateman RJ (2020) Blood plasma phosphorylated-tau isoforms track CNS change in Alzheimer's disease. J Exp Med 217:e20200861.

Biogen Inc (Press release, April 16, 2020) Biogen, Broad Institute of MIT and Harvard, Partners HealthCare Launch Consortium to Build and Share a COVID-19 Biobank. Biogen Inc., Cambridge, MA.

Blue Cross Blue Shield (2020) National Health Index. URL: https://www.bcbs.com/ the-health-of-america/health-index

Bossuyt PM (2014) Where are all the new omics-based tests? Clin Chem 60: 1256-1257.

Collins FS and Stoffels P (2020) Accelerating COVID-19 Therapeutic Interventions and Vaccines (ACTIV): an unprecedented partnership for unprecedented times. JAMA 323:2455-2457.

Corman VM, Landt O, Kaiser M, Molenkamp R, Meijer A, Chu DK, Bleicker T, Brünink S, Schneider J, Schmidt ML, et al. (2020) Detection of 2019 novel coronavirus (2019-nCoV) by real-time RT-PCR. Euro Surveill 25:2000045.

Drosten C, Günther S, Preiser W, van der Werf S, Brodt HR, Becker S, Rabenau H, Panning M, Kolesnikova L, Fouchier RA, et al. (2003) Identification of a novel coronavirus in patients with severe acute respiratory syndrome. $N$ Engl J Med 348: 1967-1976.

FDA-NIH Biomarker Working Group (2016) BEST (Biomarkers, EndpointS, and Other Tools) Resource, Food and Drug Administration, Silver Spring, MD.

Fishburn CS (April 15, 2020) COVID R\&D: Pharmas align behind crowdsourcing solution. BioCentury Inc. URL: https://www.biocentury.com/article/304928

Garrett SL, McDaniel D, Obideen M, Trammell AR, Shaw LM, Goldstein FC, and Hajjar I (2019) Racial disparity in cerebrospinal fluid amyloid and tau biomarkers and associated cutoffs for mild cognitive impairment. JAMA Netw Open 2: e1917363.

Goossens N, Nakagawa S, Sun X, and Hoshida Y (2015) Cancer biomarker discovery and validation. Transl Cancer Res 4:256-269.

Heron M (2019) Deaths: leading causes for 2017, in National Vital Statistics Reports. National Center for Health Statistics, Hyattsville, MD.

Hey SP, Gerlach CV, Dunlap G, Prasad V, and Kesselheim AS (2020) The evidence landscape in precision medicine. Sci Transl Med 12:eaaw7745.

Heymann DL (2020) Data sharing and outbreaks: best practice exemplified. Lancet 395:469-470.

Johansen M and Thomsen SF (2016) Guidelines for reporting medical research: a critical appraisal. Int Sch Res Notices 2016:1346026.

Laing AG, Lorenc A, Del Molino Del Barrio I, Das A, Fish M, Monin L, Munoz-Ruiz M, McKenzie DR, Hayday TS, Francos-Quijorna I, et al. (2020) A dynamic COVID19 immune signature includes associations with poor prognosis [published correction appears in Nat Med (2020) 26:1663]. Nat Med 26:1623-1635.

Lavezzari G and Womack AW (2016) Industry perspectives on biomarker qualification. Clin Pharmacol Ther 99:208-213.

Leptak C, Menetski JP, Wagner JA, Aubrecht J, Brady L, Brumfield M, Chin WW, Hoffmann S, Kelloff G, Lavezzari G, et al. (2017) What evidence do we need for biomarker qualification? Sci Transl Med 9:eaal4599.

Lucchini RG, Hashim D, Acquilla S, Basanets A, Bertazzi PA, Bushmanov A, Crane M, Harrison DJ, Holden W, Landrigan PJ, et al. (2017) A comparative assessment of major international disasters: the need for exposure assessment, 
systematic emergency preparedness, and lifetime health care. BMC Public Health 17:46.

Luo X, Zhou W, Yan X, Guo T, Wang B, Xia H, Ye L, Xiong J, Jiang Z, Liu Y, et al (2020) Prognostic value of C-reactive protein in patients with COVID-19. Clin Infect Dis DOI: 10.1093/cid/ciaa641 [published ahead of print].

Menetski JP, Austin CP, Brady LS, Eakin G, Leptak C, Meltzer A, and Wagner JA (2019) The FNIH biomarkers consortium embraces the BEST. Nat Rev Drug Discov 18:567-568.

Mercier T, Guldentops E, Lagrou K, and Maertens J (2018) Galactomannan, a surrogate marker for outcome in invasive aspergillosis: finally coming of age. Front Microbiol 9:661.

Misra BB, Langefeld CD, Olivier M, and Cox LA (2018) Integrated omics: tools, advances and future approaches. J Mol Endocrinol 62:R21-R45.

National Security Council (2016) Playbook for Early Response to HighConsequence Emerging Infectious Disease Threats and Biological Incidents. URL: https:/www.politico.com/news/2020/03/25/trump-coronavirus-nationalsecurity-council-149285

Park ST and Kim J (2016) Trends in next-generation sequencing and a new era for whole genome sequencing. Int Neurourol J 20 (Suppl 2):S76-S83.

Patterson TF (2011) Clinical utility and development of biomarkers in invasive aspergillosis. Trans Am Clin Climatol Assoc 122:174-183.

Petersen RC, Aisen PS, Beckett LA, Donohue MC, Gamst AC, Harvey DJ, Jack CR Jr, Jagust WJ, Shaw LM, Toga AW, et al. (2010) Alzheimer's disease neuroimaging initiative (ADNI): clinical characterization. Neurology 74:201-209.

Ponti G, Maccaferri M, Ruini C, Tomasi A, and Ozben T (2020a) Biomarkers associated with COVID-19 disease progression. Crit Rev Clin Lab Sci 57:389-399.

Ponti G, Ruini C, and Tomasi A (2020b) Homocysteine as a potential predictor of cardiovascular risk in patients with COVID-19. Med Hypotheses 143:109859.

Poste G (2011) Bring on the biomarkers. Nature 469:156-157.

Ransohoff DF and Gourlay ML (2010) Sources of bias in specimens for research about molecular markers for cancer. J Clin Oncol 28:698-704.

Saint-Aubert L, Lemoine L, Chiotis K, Leuzy A, Rodriguez-Vieitez E, and Nordberg A (2017) Tau PET imaging: present and future directions. Mol Neurodegener 12:19.
Scavone C, di Mauro G, Mascolo A, Berrino L, Rossi F, and Capuano A (2019) The new paradigms in clinical research: from early access programs to the novel therapeutic approaches for unmet medical needs. Front Pharmacol 10:111.

Sheridan C (2020) Coronavirus and the race to distribute reliable diagnostics. Nat Biotechnol 38:382-384.

Simon R (2011) Genomic biomarkers in predictive medicine: an interim analysis. EMBO Mol Med 3:429-435.

U.S. Food and Drug Administration (2020a) Emergency Use Authorization. URL: https://www.fda.gov/emergency-preparedness-and-response/mcm-legal-regulatoryand-policy-framework/emergency-use-authorization

U.S. Food and Drug Administration (2020b) List of Qualified Biomarkers. URL: https:// www.fda.gov/drugs/biomarker-qualification-program/list-qualified-biomarkers

Vandenbroucke JP (2009) STREGA, STROBE, STARD, SQUIRE, MOOSE, PRISMA GNOSIS, TREND, ORION, COREQ, QUOROM, REMARK... and CONSORT: for whom does the guideline toll? J Clin Epidemiol 62:594-596.

Weiner MW, Veitch DP, Aisen PS, Beckett LA, Cairns NJ, Green RC, Harvey D, Jack CR Jr, Jagust W, Morris JC, et al. Alzheimer's Disease Neuroimaging Initiative (2017) The Alzheimer's Disease Neuroimaging Initiative 3: continued innovation for clinical trial improvement. Alzheimers Dement 13:561-571.

World Health Organization (January 5, 2020) Pneumonia of unknown cause - China World Health Organization, Geneva, Switzerland.

Wu F, Zhao S, Yu B, Chen YM, Wang W, Song ZG, Hu Y, Tao ZW, Tian JH, Pei YY, et al. (2020) A new coronavirus associated with human respiratory disease in China. Nature 579:265-269.

Xu B and Kraemer MUG; Open COVID-19 Data Curation Group (2020) Open access epidemiological data from the COVID-19 outbreak. Lancet Infect Dis 20:534.

(2020) Science in the time of COVID-19. Nat Hum Behav 4 (4):327-328, doi: 10.1038/ s41562-020-0879-9 32303722.

Address correspondence to: Dr. Vishal S. Vaidya, Worldwide Research, Development and Medical, Pfizer Inc., 300 Technology Square Dr., 3rd Floor, Cambridge, MA 02139. E-mail: vishal.vaidya@pfizer.com 